\title{
Entwicklung eines rückführbaren, selbstkalibrierenden, absoluten TDLAS-Hygrometers in kompakter 19“ Bauweise
}

\author{
Bernhard Buchholz ${ }^{1,2}$; Norbert Böse ${ }^{1}$; Steven Wagner ${ }^{1,2}$; Volker Ebert ${ }^{1,2}$ \\ ${ }^{1}$ Physikalisch-Technische Bundesanstalt (PTB), Bundesallee 100, 38116 Braunschweig; \\ ${ }^{2}$ Center of Smart Interfaces, CSI, Technische Universität Darmstadt, Petersenstraße 32, 64287 Darmstadt; \\ corresponding author: volker.ebert@ptb.de
}

\section{Kurzzusammenfassung}

Absolutgenauigkeit, Empfindlichkeit, Langzeitstabiltät und mechanische Robustheit stellen sowohl für Industrie- als auch für Forschungsanwendungen die wichtigsten Eigenschaften von Gasanalysegeräten dar. Das neue, auf Absorptionsspektroskopie (TDLAS) basierende, diodenlasergestützte, selbstkalibrierende Hygrometer erreicht als autonomes, kompaktes (19“ $4 \mathrm{HU}$ ) Instrument bei 2.1 Sekunden Zeitauflösung eine optimale Nachweisgrenze von $33 \mathrm{ppbv}$ (normiert: $72 \mathrm{ppbv} \cdot \mathrm{m} \cdot \mathrm{Hz}^{-1 / 2}$ ). Erste Tests am nationalen Primärnormal der mittleren Gasfeuchte an der Physikalisch-Technischen Bundesanstalt ergaben absolute Abweichungen des Instruments, das selbst keine Kalibrierung an der Zielspezies benötigt, von unter $1 \%$.

\section{Einleitung}

An moderne Hygrometer, also Messgeräte zur Erfassung von gasförmigem Wasserdampf, werden z.B. bei Anwendungen in der Halbleiterindustrie, wie der Reinheitsanalyse von Prozessgasen, oder aber in der Umweltforschung, bei der Untersuchung der atmosphärischen Wasserdampfverteilung, zunehmend höhere Anforderungen hinsichtlich Empfindlichkeit, Absolutgenauigkeit, Robustheit, Wiederholbarkeit und Stabilität der Kalibrierung gegen äußere Einflüsse gestellt; durch die komplexen und fehleranfälligen Probenahmen sind z.B. Bypass-Lösungen nicht immer realisierbar. Der hohe Stellenwert der Hygrometrie ergibt sich einerseits daraus, dass Wasser durch seine starke Adsorptionsfähigkeit in vielen Prozessgasen eine nur sehr schwer zu vermeidende und daher häufig die mit Abstand größte vorliegende Verunreinigung ist. Andererseits ist Wasserdampf als stärkstes Treibhausgas von großer Bedeutung in der Atmosphäre und ein entscheidender Parameter für Simulationsmodelle der Umweltphysik, den es hinsichtlich der atmosphärischen Vertikal- und Horizontalverteilung wiederholt und präzise zu erfassen gilt. Sowohl für die Reproduzierbarkeit, Stabilität und Effizienz von Fertigungsprozessen als auch für die Vergleichbarkeit atmosphärischer Feuchtemessungen in den zunehmend globalen Messnetzen sind, neben der Robustheit, Absolutgenauigkeit und Stabilität der eingesetzten Hygrometer von größter Wichtigkeit. Dem wird gewöhnlich durch häufiges, damit aber auch kosten- und zeitaufwendiges Kalibrieren der Sensoren mit zertifizierten, im besten Fall auf SI-Standards rückführbaren Prüfgasen bzw. Transferstandards Rechnung getragen. Die Prüfgasgemische herzustellen und in Flaschen abzufüllen, ist jedoch für Wasserdampf äußerst aufwendig und hinsichtlich der Stabilität der Gasgemische mit starken Einschränkungen verbunden. Selbstkalibrierende Absolutmessverfahren 
wie die Laserhygrometrie [1], die in bestimmten Varianten auf Kalibrierung verzichten können, sind daher für den Wassernachweis hochinteressant und von großer Bedeutung [2]. Die Entwicklung neuer, rückführbarer und selbstkalibrierender Messsysteme, die auf eine Kalibrierung verzichten können, basiert im Allgemeinen auf theoretisch gut verstandenen, nachvollziehbaren und kontrollierbaren physikalischen Effekten zur Ermittlung des Messwertes.

\section{Direkte Diodenlaser-Absorptionsspektroskopie (TDLAS)}

Unsere Arbeitsgruppe hat in der Vergangenheit zahlreiche diodenlasergestützte Absorptionsspektrometer für verschiedenste Anwendungsfelder und Gasspezies entwickelt und diese unter verschiedensten Messbedingungen, wie z.B. in Labor- [3], Großforschungs- [4], Umwelt- [5] oder auch sehr rauen Industrieumgebungen [6], erfolgreich eingesetzt und validiert [7].

\section{Grundprinzip}

Das Grundprinzip der Tunable Diode Laser Absorption Spectroscopy (TDLAS) [8] und die Vorgehensweise der absoluten, selbstkalibrierenden Absorptionsspektroskopie wurde in der Literatur bereits mehrfach ausführlich erklärt [1], so dass nur auf die Besonderheiten des hier vorgestellten Spektrometers eingegangen wird. Bei der für das neue Instrument genutzten direkten TDLAS (Abb. 1) wird eine Gasprobe mit einer schmalbandigen, kontinuierlich abstimmbaren Lichtquelle (Diodenlaser) durchstrahlt, wobei eine molekülspezifische, spektral schmalbandige Abschwächung stattfindet. Distributed Feedback Diodenlaser (DFB-Diodenlaser) eignen sich wegen ihrer hohen Strahlqualität, ihrer geringen Halbwertsbreite $(\mathrm{MHz})$, ihrer hohen Seitenmodenunterdrückung $(50 \mathrm{~dB})$, ihrer meist ausreichenden Abstimmtiefe (DFB-Laser ca. $2 \mathrm{~cm}^{-1}$ ), ihrer guten Verfügbarkeit bei den erforderlichen Wellenlängen, dem einfachen Betrieb bei Raumtemperatur und ihrer allgemeinen mechanischen Robustheit dafür besonders. Bei der TDLAS wird die Laserwellenlänge zyklisch durch eine Strommodulation in Form einer Dreieckfunktion mit einer typischen, an die Anwendung anpassbaren Wiederholrate zwischen 100 und $10 \mathrm{kHz}$ moduliert und der spektrale Bereich somit periodisch hochaufgelöst abgetastet.

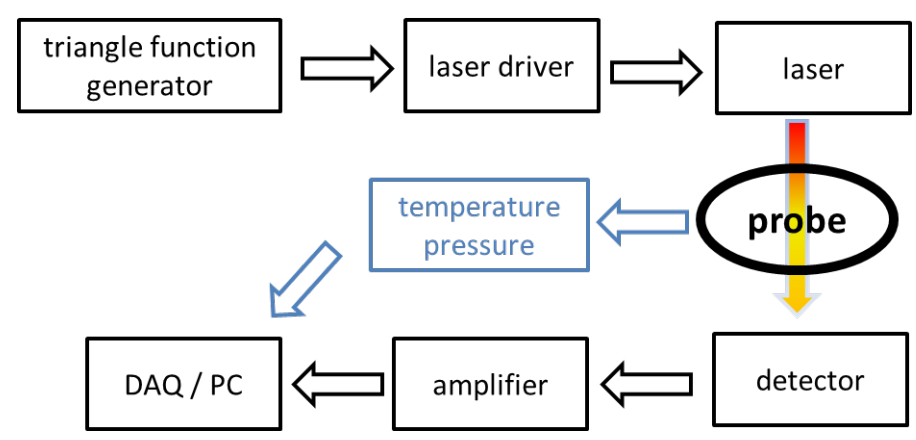

Abb. 1: Grundaufbau eines direkten TDLAS-Spektrometers

Durch Auswahl der für die Anwendung passenden [9] Absorptionslinie(n) (vgl. Abb. 2), kann die Querempfindlichkeit auf andere Gase vermindert oder sogar vermieden werden. Bei dem vorgestellten 
Gerät wurde die $110 \rightarrow 211$ Linie aus dem $1.4 \mu \mathrm{m}$-Band bei $1370 \mathrm{~nm}$ gewählt. Der Vorteil dieses Wellenlängenbereichs ist die sehr gute Verfügbarkeit und die hohe Qualität der Komponenten, da die optische Telekomunikation ebenfalls in diesem Bereich durchgeführt wird, so dass ein komplett fasergekoppelter Aufbau möglich wird, bei dem Einflüsse von parasitärem Wasser auf dem optischen Weg, der sich nicht im eigentlichen Messmedium befindet, minimiert werden können.

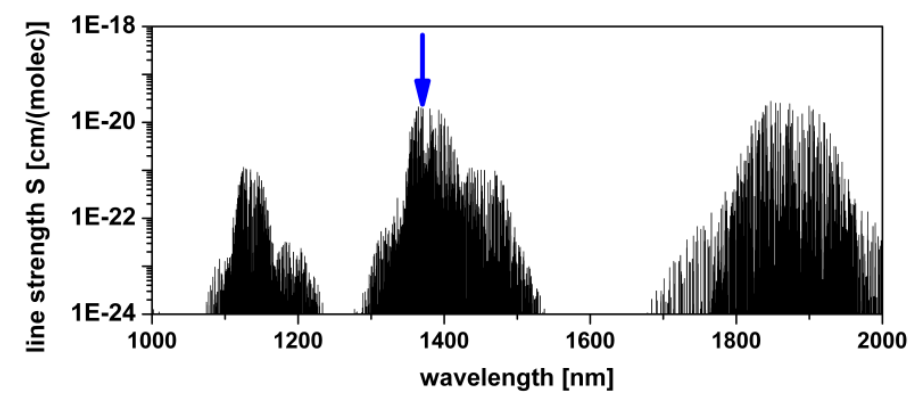

Abb. 2: Simulation (HITRAN08-Datenbank [10]) der Absoptionsbanden von Wasser im Bereich von $1000 \mathrm{~nm}$ bis $2000 \mathrm{~nm}$. Die gewählte Absorptionslinie liegt im $1.4 \mu \mathrm{m}$ Band

\section{Theorie}

Bei den geringen Lichtintensitäten, die bei der TDLAS eingesetzt werden (einige $\mathrm{mW}$ ), kann die Absorption durch das erweiterte Lambert-Beer'sche Gesetz (Eq. 1) beschrieben werden, das bspw. zur Bestimmung von absoluten Konzentrationen mit Hilfe des idealen Gasgesetzes erweitert werden kann (Eq. 2).

Eq. 1: $I(\lambda)=E(t)+I_{0}(\lambda) \cdot \operatorname{Tr}(t) \cdot \exp \left[-S(T) \cdot g\left(\lambda-\lambda_{0}\right) \cdot N \cdot L\right]$

Eq. 2: $\quad c=\frac{k_{B} \cdot T}{S(T) \cdot L \cdot p} \int \ln \left(\frac{I(v)-E(t)}{I_{0}(v) \cdot \operatorname{Tr}(t)}\right) \frac{d v}{d t} d t$

Die Gleichung beinhaltet folgende Parameter: $c$ ist die Konzentration, $k_{B}$ die Boltzmann-Konstante, $T$ die gemessene Temperatur, $L$ die feste Länge des optischen Pfades und $I(v)$ die vom Detektor gemessene Intensität nach Durchstrahlung des Mediums. Die Ausgangsintensität des Lichtes $I_{0}(v)$, die Hintergrundemission $E(t)$ und die Transmissionsverluste $\operatorname{Tr}(t)$ ergeben sich während des Auswerteprozesses direkt aus jedem einzelnen, gemessenen Rohsignal; es wird also insbesondere kein zweiter Referenzkanal oder ähnliches benötigt. Der als dynamisches Abstimmverhalten bezeichnete Faktor $\frac{d v}{d t}$ ist ein Charakteristikum des verwendeten Lasers und seiner Betriebsparameter wie Diodenstrom und Temperatur; es ist in den aktuell messbaren Unsicherheitsgrenzen eine einmal zu ermittelnde Größe, bestimmt durch die Interferenzstruktur beim Durchgang durch ein Luftetalon bestimmt [1]. Die Linenstärke $S(T)$ wird häufig der Liniendatenbank HITRAN [10] entnommen bzw. in eigenen Messungen ermittelt oder kann prinzipiell auch theoretisch berechnen werden; dies ist jedoch selbst bei Molekülen mit wenigen Atomen so komplex, dass aktuell die meisten Liniendaten experimentell bestimmt sind. Diese einzelne Größe ist allerdings geräteunabhängig und lässt sich mit völlig anderen Messmethoden, z.B. mit hochauflösenden FTIR-Geräten, messen, so dass sich nach ihrer akkurateren 
Bestimmung die Absolutgenauigkeit der Spektrometer unmittelbar verbessert. Schließlich findet sich als letzter Parameter in der Gleichung (Eq. 2) die Länge $L$ des optischen Pfades; sie wird durch die Konstruktion des Aufbaus festgelegt und stellt neben der Auswahl der passenden Absorptionslinie (Linienstärke) die zweite Möglichkeit der linearen Skalierung der Auflösung des Spektrometers dar.

Das Entscheidende an dieser Gleichung (Eq. 2) ist, dass in sie keine weiteren Parameter eingehen, die der Kalibrierung bedürfen. Druck- und Temperatursensoren sind zu kalibrieren, was mit sehr hoher Genauigkeit vorgenommen werden kann (Druckunsicherheit $<10^{-4}$ (full scale), Temperaturunsicherheit $<0,1 \mathrm{~K}$ ). Höherwertige Sensoren sind in diesem Bereich auch langzeitstabil, so dass aktuell ihr relativer Einfluss im Fehlerbudget gering ist. Auf eine Kalibrierung am zu messenden Zielgas kann daher verzichtet werden, oder aber sie wird zur Erhöhung der Absolutgenauigkeit vorgenommen, so dass das Spektrometer in zwei Modi betreibbar ist.

\section{Auswerteprozess}

Die vom Detektor nach Durchstrahlung der Probe erfasste Intensität $I(v)$ wird digitalisiert und dann mit der von der Arbeitgruppe entwickelten interaktiven Software ausgewertet. In vereinfachter Darstellung (Details [1]) wird dazu das Rohsignal mittels des oben beschriebenen dynamischen Tuning-Faktors in den Wellenzahlraum transferiert. An dieses Profil (Abb. 3) wird dann eine Formfunktion angepasst (Levenberg-Marquardt-Algorithmus), die sich in den hier vorgestellten Messungen aus einem Polynom für die Basislinie und einem Voigt-Profil, einer Faltung aus Lorentz-Profil (Druckverbreiterung) und GaußProfil (Temperaturverbreiterung) für die eigentliche Absorptionslinie zusammensetzt. Je nach Güte des Signals und der konkreten Anwendung kann zur Minimierung der Freiheitsgrade aus Druck und Temperatur die Verbreiterung des Voigt-Profils und die relative Position der Absorptionslinie vor dem Fitprozess berechnet werden.

Die nach der eigentlichen Messung durchführbaren Auswertungen ermöglichen, auch bei einer sehr schnellen Abstimmung des Lasers und bei gleichzeitiger schneller Messung von Druck und Temperatur, jede dieser einzelnen Perioden individuell und hochaufgelöst auszuwerten. Da jedes einzelne Rohsignal (Abb. 3) die gesamte Information enthält, ist eine Zeitauflösung im Millisekunden-Bereich möglich, wie z.B. in [6] demonstriert wurde.

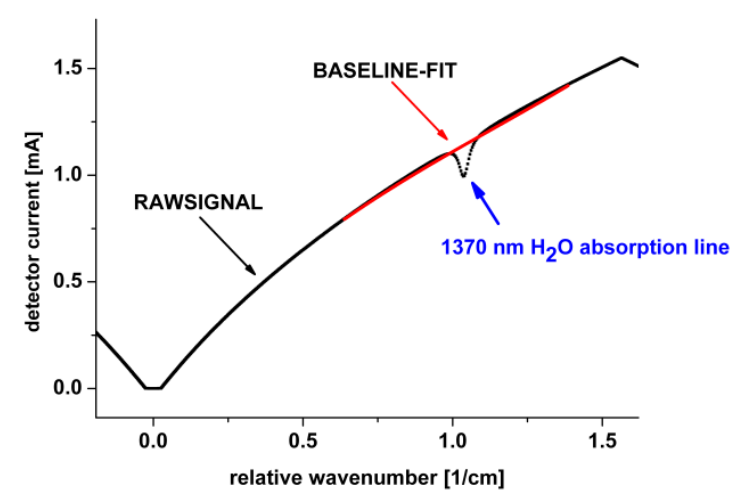

Abb. 3: Typisches Rohsignal einer Messung bei mittlerer Wasserdampf-Konzentration (ca. 1000 ppmv); das Absorptionssignal ist sehr gut sichtbar. Der Laserstrom wird mit einer Dreieckform moduliert. Die Krümmung der Kurve ergibt sich durch Umrechnung in den Wellenzahlraum, denn der Zusammenhang zwischen Abstimmtiefe und Strommodulation ist nichtlinear [1]. 


\section{Instrument}

Bei der vorgestellten Entwicklung des Laserhygrometers "SEALDH“ (Selective Extractive Airborne Laser Diode Hygrometer) handelt es sich um ein extraktiv messendes, selbstkalibriendes, absolutes, speziesselektives und, vor allem, rückführbares Hygrometer auf der Grundlage direkter TDLAS (Tunable Diode Laser Absorption Spectroscopy) bei $1.4 \mu \mathrm{m}$ Laserwellenlänge. Das Gerät, das sich während des Betriebes komplett selbst überwacht und bei Störungen ggf. auch neu startet, ist als autonomes System in kompakter 19“ Bauweise (4HE) konzipiert. Es basiert auf einem Mikrorechner mit Atom-Prozessor und Windows XP Standard-Betriebssystem, was die einfache Fernkontrolle über kabel- oder funkgestützte Datennetze ermöglicht. Dank der geringen Stromaufnahme von < 90 Watt und der allgemeinen Erschütterungsunempfindlichkeit der TDLAS eignet es sich sowohl für industrielle Feldanwendungen wie auch für umweltphysikalische Einsätze bspw. auf Forschungsflugzeugen. Die Optik ist komplett fasergekoppelt, die Messzelle in White-Konfiguration (Zellvolumen $300 \mathrm{~cm}^{3}$ ) erlaubt eine einstellbare optische Pfadlänge von $0.15 \mathrm{~m}$ bis $1.5 \mathrm{~m}$ und somit eine einfache, mit den häufig eingesetzten Taupunktspiegeln nicht mögliche Skalierung des Messbereiches; eine Ankoppelmöglichkeit für eine offene in-situ-Messstrecke ist ebenfalls vorhanden. Die Wiederholrate des vorgestellten Hygrometers beträgt $140 \mathrm{~Hz}$ und gestattet eine maximale Zeitauflösung von $7 \mathrm{~ms}$. Die Ausstauschrate des Gases limitiert bei extraktiven Messungen somit die Antwortzeit des Gerätes. Bei einer optischen Pfadlänge von $\mathrm{L}=1.5 \mathrm{~m}$ und einem Druckbereich zwischen 0 und $1500 \mathrm{hPa}$ liegt der Dynamikbereich des SEALDHHygrometers bei 50 bis 25000 ppmv $\mathrm{H}_{2} \mathrm{O}$, da es aktuell primär für die flugzeugbasierte Messung in der Troposphäre eingesetzt wird.
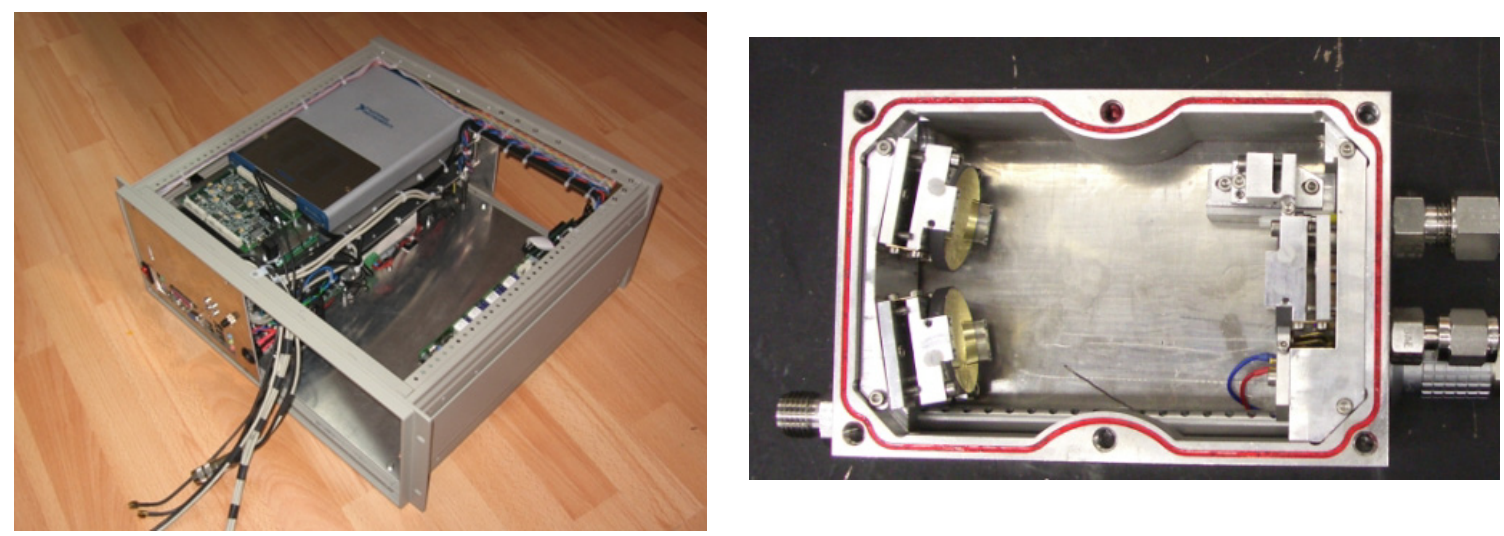

Abb. 4: Links: Kompakte Version des Spektrometers (19" 4HE Standard Rack) in stand-alone-Ausführung. Der Laser und die extraktive Zelle wurden entfernt, um einen besseren Blick auf die Elektronik zu erhalten.

Rechts: Optische Messzelle in White Konfiguration mit eingebautem Thermoelement und Detektor. Die Abmessungen betragen $120 \mathrm{~mm}$ × $80 \mathrm{~mm}$, das Zellvolumen $300 \mathrm{~cm}^{3}$, die optische Pfadlänge ist bis zu $1.50 \mathrm{Meter}$ einstellbar.

\section{Optische Kurzzeit-Pråzision}

Um die optische Kurzzeit-Präzision zu untersuchen, sind zwei bei $140 \mathrm{~Hz}$ Repetitionsrate aufgenommene, nicht gemittelte Messsignale mit ihren angepassten Voigt-Profilen gezeichnet. Bei niedriger Konzentration (Abb. 5) wurde eine maximale optische Dichte $O_{D_{\text {Peak }}}$ von $7.23 \times 10^{-2}$ ermittelt, so dass sich, mit einem Residuum von $2.96 \times 10^{-4}(1 \sigma)$, ein Signal-zu-Rauschverhältnis von 244 ergibt. 
Ausgehend von der zugehörigen Konzentration von 600 ppmv ergibt sich damit eine Präzision von $2.45 \mathrm{ppmv}$ (normierte Präzision : $0.31 \mathrm{ppmv} \cdot \mathrm{m} \cdot \mathrm{Hz}^{-\frac{1}{2}}$ ).

Bei hohen Konzentrationen (Abb. 6) ist im Residuum eine Reststruktur sichtbar, die daraus resultiert, dass die Anpassung der Profile an die Messdaten mit aus Druck und Temperatur vorberechneter Breite (Fremd- und Eigenverbreiterung) zur Minimierung der Freiheitsgrade erfolgt. Die Daten, die zu dieser Linie in HITRAN hinterlegt sind, haben eine Unsicherheit von $5-10 \%$, so dass diese Effekte bei hohem Signal-zu-Rauschverhältnis sichtbar werden. Aktuell in Planung ist es, diese Linienparameter in der Physikalisch-Technischen Bundesanstalt hoch präzise zu vermessen mit dem Ziel, akkuratere Daten zu erhalten. Um von der Reststruktur zu abstrahieren, ist das lokale Residuum zu $3.2 \times 10^{-4}(1 \sigma)$ bestimmt, was bei einer maximalen optischen Dichte $\mathrm{OD}_{\text {Peak }}$ von $4.61 \times 10^{-1} \mathrm{zu}$ einem Signal-zu-Rauschverhältnis von 1440 führt. Bei der Konzentration von 3920 ppmv ergibt sich eine Präzision von 2.72 ppmv (normierte Präzision : $0.34 \mathrm{ppmv} \cdot \mathrm{m} \cdot \mathrm{Hz}^{-\frac{1}{2}}$ ).

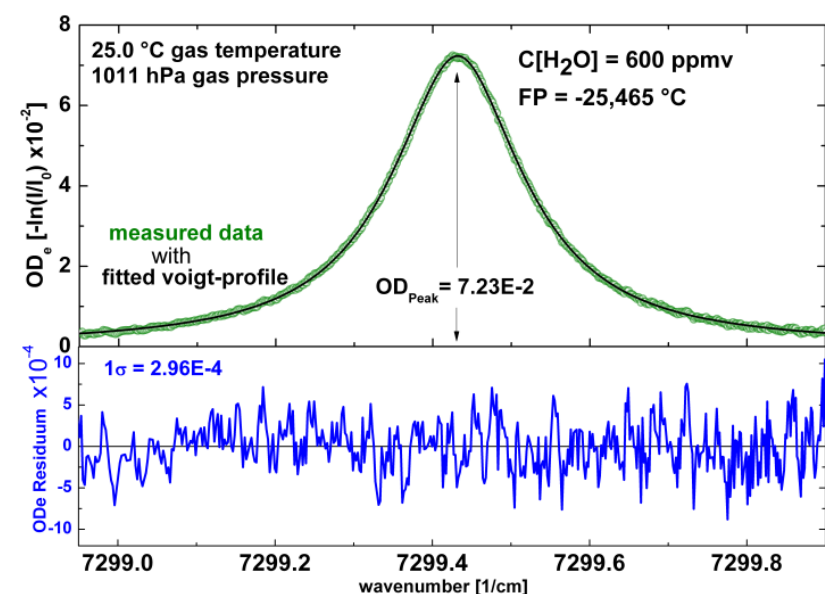

Abb. 5: Messdaten und angepasstes Voigt-Profil bei 600 ppmv Wasserdampf. Aus der optischen Dichte $O D_{\text {Peak }}\left(7.23 \times 10^{-2}\right)$ und dem Residuum $\left(2.96 \times 10^{-4}(1 \sigma)\right)$ ergibt sich ein Signal-zu-Rauschverhältnis von 244 und somit eine Präzision von 2.45 ppmv (normiert: $0.31 p p m v \cdot m \cdot H z^{-1 / 2}$ )

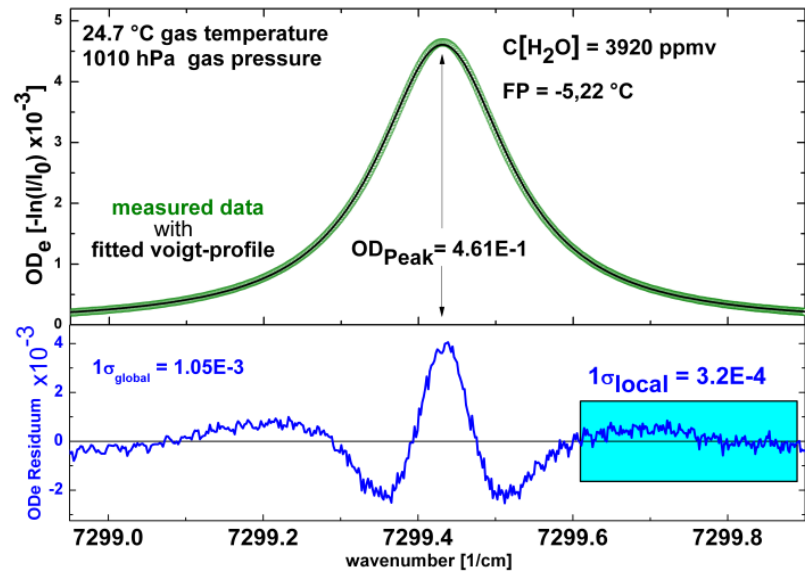

Abb. 6: Wie in Abb. 5 sind Messdaten und Voigt-Profil dargestellt. Die Breite der Linie wird mit den Liniendaten der HITRAN08-Datenbank berechnet (Details siehe Text). Wegen deren Unsicherheiten (5-10\%) zeichnet sich bei dem hohen Signal-zu-Rauschverhältnis von 1440 eine Reststruktur im Residuum ab.

\section{Optische Langzeit-Präzision}

Um das Spektrometer auf seine Langzeiteigenschaften $\mathrm{zu}$ untersuchen, wurde es an einen Feuchtegenerator der PTB angeschlossen. Dieser erzeugt nach dem Zweidruckverfahren eine konstante Wasserdampfkonzentration in Luft, die vom SEALDH-Instrument gemessen wurde. Die Messung ergab bei einem Druck von $1012 \mathrm{hPa}$ und einer Temperatur von $24.96{ }^{\circ} \mathrm{C}$ eine Konzentration von $599.04 \mathrm{ppmv}$ bei einer Standardabweichung von $0.34 \mathrm{ppmv}$. Mit diesen Ausgangsdaten wurde aus der Allan-Varianz [11] die ideale Mittelungsanzahl bestimmt. Bei einer zeitlichen Auflösung von 2.1 Sekunden ergibt sich daraus eine Präzision von 0.033 ppmv. Die normierte Präzision errechnet sich damit zu $0.072 \mathrm{ppmv} \cdot \mathrm{m} \cdot \mathrm{Hz}^{-1 / 2}$. Dass diese unter dem Wert der optischen Kurzzeit-Präzision liegt, resultiert aus den stabilen Interferenzstrukturen (vgl. Residuum Abb. 5), die von der Optik der White-Zelle kommen und daher zwar die Standardabweichung des Residuums erhöhen, aber im Zeitverlauf nicht variieren. Die Präzision in Abhängigkeit von der Systemantwortzeit kann ebenfalls aus der Allan-Varianz berechnet 
werden (Abb. 7) und ermöglicht, mittels der geforderten Antwortzeit bzw. Präzision für jeden Einsatzzweck die passende Mittelungsanzahl festzulegen.

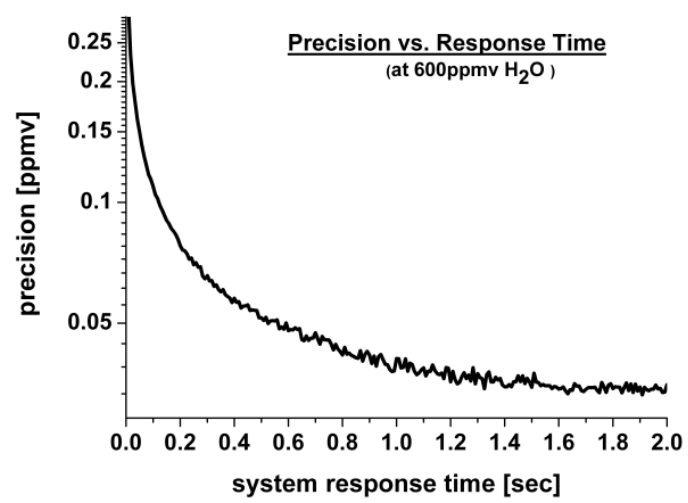

Abb. 7: Antwortzeit des SEALDH-Spektrometers gegen die dabei erreichbare Präzision. Der Graph ist eine abgewandelte Darstellung der Allan-Varianz, die zur Langzeitcharakterisierung des Spektrometers eingesetzt wurde, um die ideale Mittelungsanzahl zu bestimmen.

\section{Absolutgenauigkeit}

Erste Tests zur Absolutgenauigkeit des Spektrometers wurden am nationalen Primärnormal der Gasfeuchte, einem Zweidruck-Feuchtegenerator, an der Physikalisch-Technischen Bundesanstalt in Braunschweig durchgeführt. Ohne jede Kalibrierung des Laserhygrometers wurde der vom Normal vorgegebene Wert von $(4000 \pm 7)$ ppmv $(k=2)$ bei Standarddruck und Raumtemperatur mit einer Relativabweichung von unter $1 \%$ wiedergegeben (Abb. 8). Die Unsicherheit des SEALDH-Instruments beträgt $4 \%$ und ergibt sich vor allem (ca. $80 \%$ ) aus den spektrometrischen Unsicherheiten der Hitran Liniendaten; weitere Einflüsse sind Temperatur- und Druckunsicherheiten (6 \%).

Derzeit an der PTB anlaufende Aktivitäten zur rückgeführten und präzisen Bestimmung von Spektraldaten haben zum Ziel, die spektrometrischen Unsicherheiten deutlich zu reduzieren.
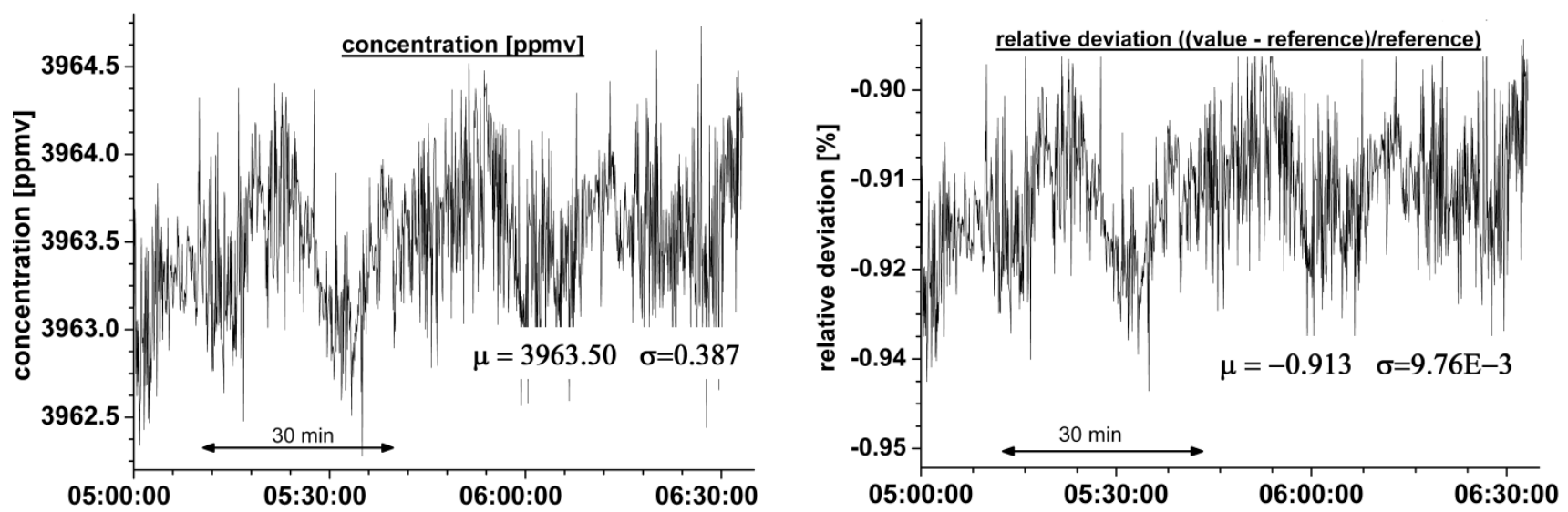

Abb. 8: Erster Test eines rückführbaren, selbstkalibrierenden Laser-Hygrometers am nationalen Feuchtenormal der PTB; dabei wurde eine Konzentration von $(4000 \pm 7)$ ppmv bei Standarddruck und Raumtemperatur vorgelegt. Die erkennbaren langsamen Schwankungen resultieren aus Schwankungen der Sättiger-Temperatur des Generators im Bereich weniger Millikelvin. Wichtig hierbei ist, dass das vorgestellte Spektrometer nicht an einem Feuchtegenerator kalibriert wurde, sondern den absoluten Messwert direkt und selbstkalibrierend aus dem Messsignal berechnet. 


\section{Zusammenfassung und Ausblick}

Die Diodenlaserhygrometrie eröffnet als selbstkalibrierendes Absolutmessverfahren die Möglichkeit, Absolutgenauigkeit, Präzision und Stabilität von Hygrometern mit typischen TDLAS Vorteilen wie Robustheit und einfachem, kostengünstigen Betrieb zu kombinieren. Das vorgestellte SEALDH-Hygrometer ist ein wichtiger Schritt in diese Richtung; es ist kompakt (19“, 4HE), erreicht aktuell eine normierte Präzision von $0.072 \mathrm{ppmv} \cdot \mathrm{m} \cdot \mathrm{Hz}^{-1 / 2}$ und ermöglicht in der bestehenden Konfiguration bei $2.1 \mathrm{~s}$ Antwortzeit eine Präzision von 33 ppbv. Ein erster Absolutvergleich mit dem nationalen Feuchtenormal der PTB ergab ohne jede Kalibrierung des TDL-Hygrometers bei ca. $1000 \mathrm{hPa}$ eine Relativabweichung von unter $1 \%$ zum vorgegebenen Wert von $(4000 \pm 7)$ ppmv ( $k=2)$. Weitere Messreihen zur vollständigen Validierung in verschiedenen Konzentrations- und Druckbereichen sind derzeit in Vorbereitung. Ein anspruchvoller Feldvergleich wurde bereits realisiert: Das Messgerät wurde in einen LEARJET 35A integriert und mit mehreren verschiedenen, etablierten Flugzeughygrometern verschiedener Messprinzipen einem Blindvergleich unterzogen. Die ersten Ergebnisse sehen sehr vielversprechend aus. Das vollständige Ergebniss wird in 2012 an anderer Stelle veröffentlicht werden [Buchholz et al, Appl.Phys. B, 2012].

\section{Literaturreferenzen:}

[1] V. Ebert and J. Wolfrum, "Absorption spectroscopy," in OPTICAL MEASUREMENTS-Techniques and Applications, ed. F. Mayinger, Springer, 1994, pp. 273-312.

[2] B. J. Murray et al., "Heterogeneous nucleation of ice particles on glassy aerosols under cirrus conditions," Nature Geoscience, vol. 3, no. 4, pp. 233-237, Mar. 2010.

[3] S. Hunsmann, K. Wunderle, S. Wagner, U. Rascher, U. Schurr, and V. Ebert, "Absolute, high resolution water transpiration rate measurements on single plant leaves via tunable diode laser absorption spectroscopy (TDLAS) at $1.37 \mu \mathrm{m}, "$ Applied Physics B, vol. 92, no. 3, pp. 393-401, Jul. 2008.

[4] V. Ebert, H. Teichert, C. Giesemann, H. Saathoff, and U. Schurath, "Fibre-Coupled In-situ Laser Absorption Spectrometer for the Selective Detection of Water Vapour Traces down to the ppbLevel," tm - Technisches Messen, vol. 72, pp. 023 - 030, 2005.

[5] W. Gurlit et al., "Lightweight diode laser spectrometer ' CHILD ' for balloon- borne measurements of water vapor and methane," Applied Optics, vol. 44, no. 1, pp. 91-102, 2005.

[6] H. Teichert, T. Fernholz, and V. Ebert, "Simultaneous in situ measurement of $\mathrm{CO}, \mathrm{H} 2 \mathrm{O}$, and gas temperatures in a full-sized coal-fired power plant by near-infrared diode lasers.," Applied optics, vol. 42, no. 12, pp. 2043-51, Apr. 2003.

[7] D. Fahey and R. Gao, "Summary of the AquaVIT Water Vapor Intercomparison: Static Experiments," source: https://aquavit. icg. kfa-juelich. de/WhitePape, no. October, 2009.

[8] C. Schulz, A. Dreizler, V. Ebert, and J. Wolfrum, "Combustion Diagnostics," in Springer Handbook of Experimental Fluid Dynamics, Editors C. Tropea, J. Foss, A. Yarin, 2007, pp. 1241-1316.

[9] K. Wunderle, T. Fernholz, and V. Ebert, "Selektion optimaler Absorptionslinien für abstimmbare Laserabsorptionsspektrometer," VDI-Berichte, vol. 1959, pp. 137-148, 2006. 
[10] L. S. Rothman et al., "The HITRAN 2008 molecular spectroscopic database," Journal of Quantitative Spectroscopy and Radiative Transfer, vol. 110, no. 9-10, pp. 533-572, Jun. 2009.

[11] D. W. Allan, "Statistics of atomic frequency standards," Proceedings of the IEEE, vol. 54, no. 2, pp. 221-230, 1966. 